\title{
Molecular Dynamics Analysis of Glucose Oxidase Stability against Temperature
}

\author{
Laksmi Ambarsari 1** (D), Farhan Azhwin Maulana 1(D), Setyanto Tri Wahyudi ${ }^{2}{ }^{\mathbb{D}}$, \\ Popi Asri Kurniatin 1(D), Waras Nurcholis ${ }^{1,3}$ (D)
}

1 Department of Biochemistry, Bogor Agricultural University, Bogor, Indonesia; laksmi@apps.ipb.ac.id (L.A.); azhwinfarhan@gmail.com (F.A.M.); popi_asri@apps.ipb.ac.id (P.A.K);

2 Department of Physics, Bogor Agricultural University, Bogor, Indonesia; stwahyudi@apps.ipb.ac.id (S.T.W.);

3 Tropical Biopharmaca Research Center, Bogor Agricultural University, Bogor, Indonesia; wnurcholis@apps.ipb.ac.id (W.N.);

* Correspondence: laksmi@apps.ipb.ac.id (L.A.);

Scopus Author ID 55903475100

Received: 26.05.2021; Revised: 5.07.2021; Accepted: 10.07.2021; Published: 13.08.2021

\begin{abstract}
Glucose oxidase (GOD) from local isolated Aspergillus niger IPBCC.08.610 shows a widespread application, specifically as a bioanode in glucose-based biofuel cells. Enzymes with adequate thermal stability are necessary for enhancing product efficiency. Also, evaluating the structural dynamics to improve temperature helps to determine the residue. The molecular dynamics simulation of GOD_IPBCC_1CF3 at temperatures of 300, 400, and $500 \mathrm{~K}$ was carried out to analyze important amino acid residues for thermal stability. The results showed that the amino acid residues responsible for thermal stability were dispersed into several essential regions, including D576 at the C terminal, E266-R250, and E38-R237 in the FAD-binding domain E485-R470 in the substrate-binding antiparallel beta system. However, the FAD molecular flexibility against temperature depends on conserve E48 by stabilizing the ribose sugar moiety.
\end{abstract}

Keywords: Glucose oxidase; Aspergillus niger IPBCC.08.610; temperature stability; molecular dynamics.

(C) 2021 by the authors. This article is an open-access article distributed under the terms and conditions of the Creative Commons Attribution (CC BY) license (https://creativecommons.org/licenses/by/4.0/).

\section{Introduction}

Glucose oxidase ( $\beta$-D-glucose:oxygen 1-oxidoreductase, EC 1.1.3.4) is a flavoenzyme that catalyzes $\beta$-D-glucose oxidation reaction using molecular oxygen as an electron acceptor to produce $\beta$-D-glucano-1,5-lactone and hydrogen peroxide. The enzyme is a homodimer with two identical subunits measuring $80 \mathrm{kDa}$. In addition, glucose oxidase possesses FAD (flavin adenine dinucleotide) is a coenzyme that is non-covalently bonded to each subunit's active site and serves as an electron carrier during catalytic reaction $[1,2]$.

The Glucose oxidase from the local isolate culture of Aspergillus niger IPBCC.08.610 (GOD_IPBCC), shows a specific activity of 773,269 $\pm 24,504 \mathrm{U} / \mathrm{mg}$ after purification. Enzyme stability testing at optimum temperature and $\mathrm{pH}\left(30^{\circ} \mathrm{C}, \mathrm{pH} 6\right)$ showed that glucose oxidase possibly maintains $50 \%$ of its total activity up to 23 minutes after the treatment [3]. Previous research reported the potential use of GOD_IPBCC as a biosensor with a $\mathrm{Km}$ and sensitivity values of $56.4 \mathrm{mg} / \mathrm{dL}$ and $1.09 \mathrm{~mA} / \mathrm{Mm}$, respectively [4]. Furthermore, as a bioelectrode at the MCPE anode, the enzyme generated activation energy, maximum current, and sensitivity of $16.4 \mathrm{~kJ} \mathrm{~mol}^{-1}, 3.95 \mathrm{~mA}$, and $7.79 \mathrm{~mA} \mathrm{mM}^{-1} \mathrm{~cm}^{-2}$, correspondingly [5]. 
The enzyme stability required to operate effectively in non-physiological conditions $(\mathrm{pH}$ and extreme temperatures) is a significant consideration in terms of appropriately catalyzing substrates and an opportunity for further applications. Therefore, the present study analyzes the structural dynamics of GOD_IPBCC using molecular dynamic simulation with increasing temperatures to determine the residues responsible for the thermostability. The prediction GOD_IPBCC structure was previously investigated by homology modeling resulting in GOD_IPBCC_1CF3, which is used as a template in this present study [6].

\section{Materials and Methods}

\subsection{Materials.}

This research involves the use of three-dimensional GOD structure as the primary sample based on amino acid sequence from NCBI (accession number: MH593586) [7], with SWISSMODEL application [8].

\subsection{Tools.}

The applied tools are categorized into two aspects, termed hardware and software. Hardware refers to a set of personal computers with Quad-Core Processor (Intel CoreI7), 8 gigabytes of RAM, Graphic Card NVIDIA GeForce GTS 940032 core GPU, and LINUX Ubuntu operating system, version 16.04 LTS. Meanwhile, the software for MD simulation incorporates the assisted model building with energy refinement (AMBER), version 16 package [9]. GOD_IPBCC residual alignment visualization was performed using the coffee web server page [10]. Subsequently, the protonated state of the protein constituent side chains was adjusted with the $\mathrm{H}++$ device [11], while the amino acid characteristics were calculated with the ProtParam program [12]. This step was followed by the structural validation analysis of the modeling results with the use of PROCHECK [13], PDBsum [14], molprobity [15], as well as proQ [16], and the simulation results were evaluated with cpptraj in ambertools16 package [17]. Furthermore, the affinity energy was calculated by molecular mechanics poison Boltzmann and surface area (MMPBSA) [18], while the beta factor value of GOD_IPCC structure was obtained from the B-fitter program [19]. The prediction of protein functional areas was performed with the Pfam web server [20]. Visual molecular dynamics (VMD) version 1.9.2 [21] and PyMOL (PyMOL molecular graphics system, version 1.8 Schrodinger, LLC) were used for protein structure visualization. Meanwhile, Ms. Excel 2010, and Gnuplot version 4.6 were employed in data processing and graph plotting, respectively.

\subsection{System preparation and minimization.}

System preparation and minimization were conducted depending on the modified procedure of Case et al., [9]. In system preparation, hydrogen atoms and other organic molecules protein (pdb files) were initially removed using the ambpdb program. Subsequently, a new hydrogen atom was added, and the protonated state on each residue side chain was also adjusted. The output is a topology file and coordinate, converted into pdb files with ambpdb in the AMBER packet system. Also, system preparation was performed using the ff14SB force field in an explicit solvent, where the resulting protein structure was solvated by implementing the TIP3P water molecule, with a box size of 16 Á. Figure 1a shows the addition of sodium and chlorine ions to neutralize the system running on Leap in each input file (with and without 
boxes). This phenomenon produces topology, coordinates, and pdb files [9]. Meanwhile, in system minimization, the resulting topology and coordinate files are then prepared for constrained minimization. The method is categorized into 7 separate script files, and for each unit, the hydrogen atoms are constrained and gradually released. Structural minimization calculations were performed using the steepest descent and conjugate gradient algorithms in a total of 110,000 steps. The minimized output is evaluated using the mdout analyzer, and the output generates a new coordinate file for the molecular dynamics system [9].

\subsection{Molecular dynamics simulation.}

Molecular dynamics simulations were performed in line with the Case et al., [9], involving several stages, including system heating and equilibration, production simulation, results in analysis, and binding energy calculations.

\subsubsection{System heating and equilibration.}

The system was gradually heated to $300 \mathrm{~K}$ with incremental intervals of $50 \mathrm{~K}$ on 6 separate script files (sample script in Figure 2a). A langevin thermostat in the NVT ensemble (fixed volume and temperature) was implemented. The equilibrium transition switches to an NPT system (fixed pressure and temperature) for $50 \mathrm{ps}$, as the system approaches $300 \mathrm{~K}$. However, the constraints from the heating stage were steadily released slowly, resulting to free naturally moving proteins. The equilibration was performed by creating sample input files. Examination of the system heating and the results were evaluated similarly to the minimization step by checking the temperature and molecular density [9].

\subsubsection{Production simulation.}

This process generated a significant amount of solvated proteins from the final equilibration (in NPT ensembles) for $50 \mathrm{~ns}$ in 25 separate script files (each script for $2 \mathrm{~ns}$ ). The trajectories were stored at every 2000 steps, and the non-bonding cutoff value of $10 \AA$ was applied. Furthermore, the Particle Mesh Ewald (PME) algorithm was performed to calculate the electrostatic energy of the protein. Figure 2 shows an example script for a production simulation. The overall MD procedure was also aimed at modeling the thermostability of the holo and apo-shaped structures with the heating temperature increased to 350,400 , and $500 \mathrm{~K}$ [9].

\subsubsection{Analysis of simulation results and calculation of binding energy.}

Trajectories in netcdf format from production simulations were combined into a single file to remove the sodium salt and solvent, leaving the protein without a box. The MD analysis included RMSD, RMSF, non-bonding energy, solvent accessibility (SASA), secondary structure changes, salt bridges, hydrogen bonds, and structural visualization of holo and apo forms. Also, the resulting hydrogen bond was calculated at a distance between the donor and acceptor atoms of $3 \AA$, while for the salt bridge, the cutoff distance value of oxygen and nitrogen atoms was estimated at $3.2 \AA$. However, the surface area of the three catalytic triads residue was determined by calculating the arbitrary triangle area for each increase in system temperature. Meanwhile, the binding affinity between the enzyme and coenzyme was analyzed by evaluating the molecular mechanics of Poisson Boltzmann/generalized Born surface area (MMPB/GBSA). Three separate file components are needed during this stage, including 
complex topology files, receptors, and ligands generated using the ante_MMPBSA.py program [18]. These three files and the combined trajectories for each structure were executed at $10 \mathrm{ps}$ intervals, with a salt concentration of $100 \mathrm{mM}$.

\section{Results and Discussion}

\subsection{Stabilizer residue through structural thermostability analysis.}

Molecular dynamics simulations were performed on the GOD_IPBCC_1CF3 structure of both apoenzyme and holoenzyme (enzymes with FAD as a coenzyme). Figure 1 shows the use of three temperature variations, 350,400 , and $500 \mathrm{~K}$, in determining the thermostable enzyme region. Based on observations, applying heat at $350 \mathrm{~K}$ for $50 \mathrm{~ns}$ generated a lesser holoenzyme RMSD value compared to the apoenzyme RMSD. This indicates a more stable structure, although the stability for both materials remains useful. Meanwhile, at 400K, similar RMSD values were observed for the two structures, with continuous elevation. In addition, an increase of approximately $0.3 \AA$ indicates a gradual denaturation process at this temperature. These results are in line with Ambarsari et al., (2016), where the thermal stability of commercial GOD derived from A. niger in micro fuel cell applications is able to attain $70^{\circ} \mathrm{C}$ (343 K) as observed by the largest current strength value [5]. Figure 1a shows a similar condition at $500 \mathrm{~K}$, where both apoenzyme and holoenzyme RMSD values continued to expand. However, the GOD_IPBCC_1CF3 structure steadily becomes unstable, but structurally (alpha-helix and beta) as the interior remains intact despite a conformational change. This is indicated by the high RMSD value extending to $8 \AA$ (holoenzyme structure) (Figure 1a). The RMSD value represents enzyme instability [22-24], where higher estimates generate a more unstable structure. Furthermore, the increase in structural destabilizing properties in holoenzyme is proportional to the high RMSD in its coenzyme. This effect is probably due to more unstable structural components in FAD than other segments, leading to a decline in noncovalent interactions between these components and surrounding residues.

\section{Apoenzim Holoenzim}
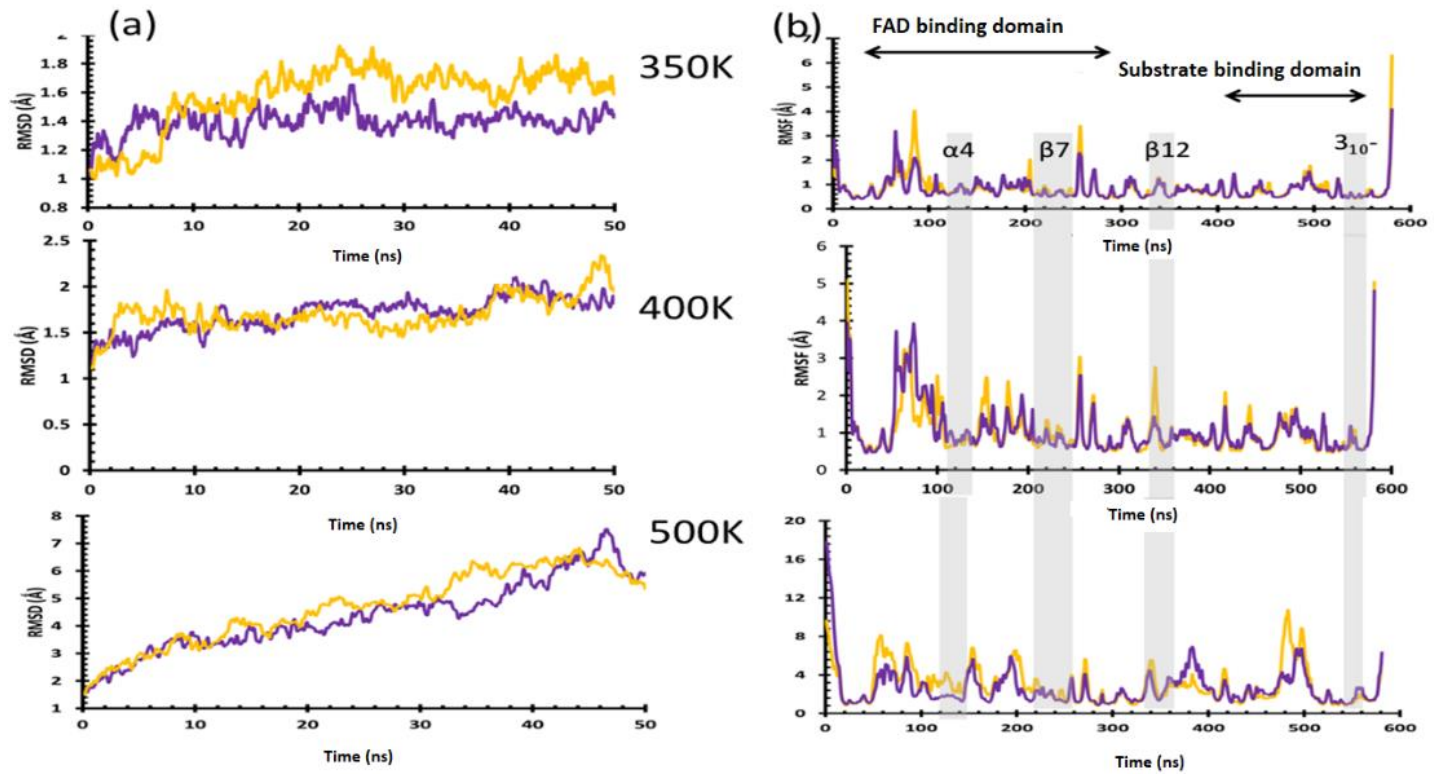

Figure 1. (a) RMSD curves for C-alpha atoms; (b) RMSF per residue as a function of thermal stability simulation time in the two structural forms of GOD_IPBCC_1CF3. The stabilizer residue is shown with a gray shaded line. 
Another observed parameter refers to the RMSF value. During the simulation, RMSF analysis investigates residual fluctuations and flexibility [25-27]. The results showed that conserved residues were present in the heating treatment $(350,400$, and $500 \mathrm{~K})$ as well as in several domains (Figure 1b). The particles include FAD-binding domain (125-140, 230-250), inter-domain (320-340), and substrate-binding domain residues (540-562). These components sequentially form alpha helix ( $\alpha 4$ ), beta-sheet ( $\beta 7$ and 12), and $3, \neg 10$-helical structures. Also, the heating treatment produces residues that form loops, as indicated by residues in 150-156 and 380-390 ranges and the hypersensitive region of the protein core (210-215). Figure 1b shows the residues are indicated by a significant increase in the RMSF value per particle. The component without a conformational change in the secondary structure or conserved residue is considered to exhibit structurally thermostable characteristics based on the treatment data.
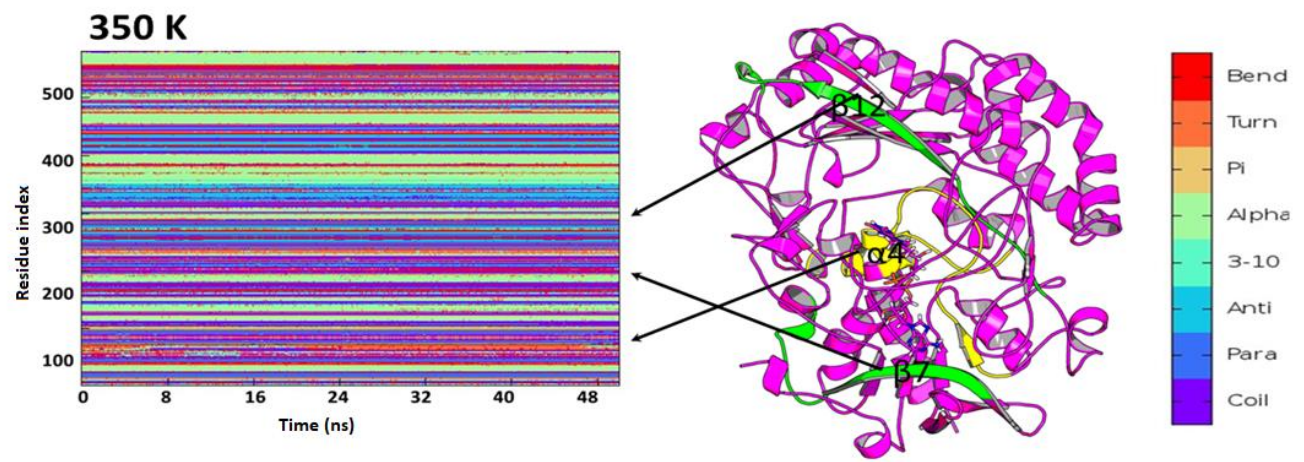

$400 \mathrm{~K}$
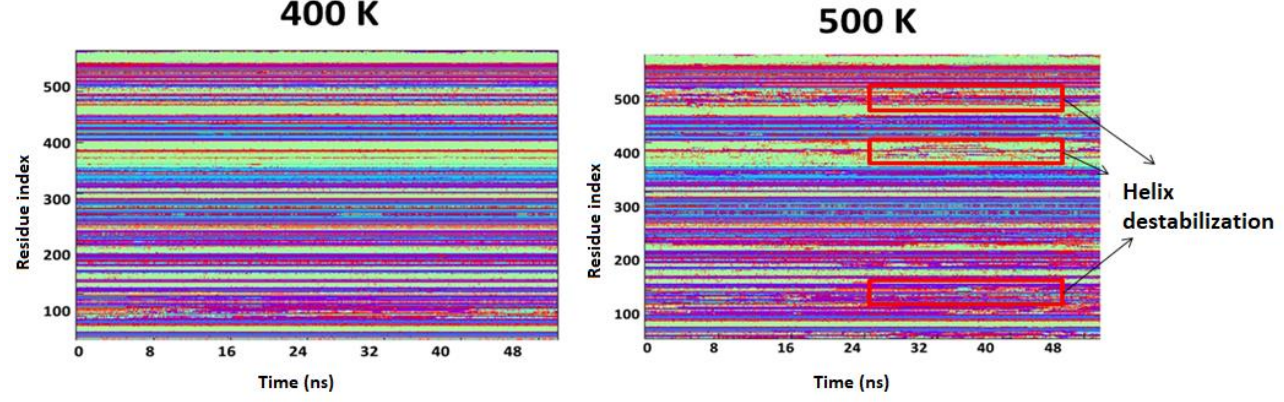

Figure 2. The final trajectory visualization at $350 \mathrm{~K}$ of GOD_IPBCC_1CF3 and secondary structure changes plotted as a residual function for each temperature increase.
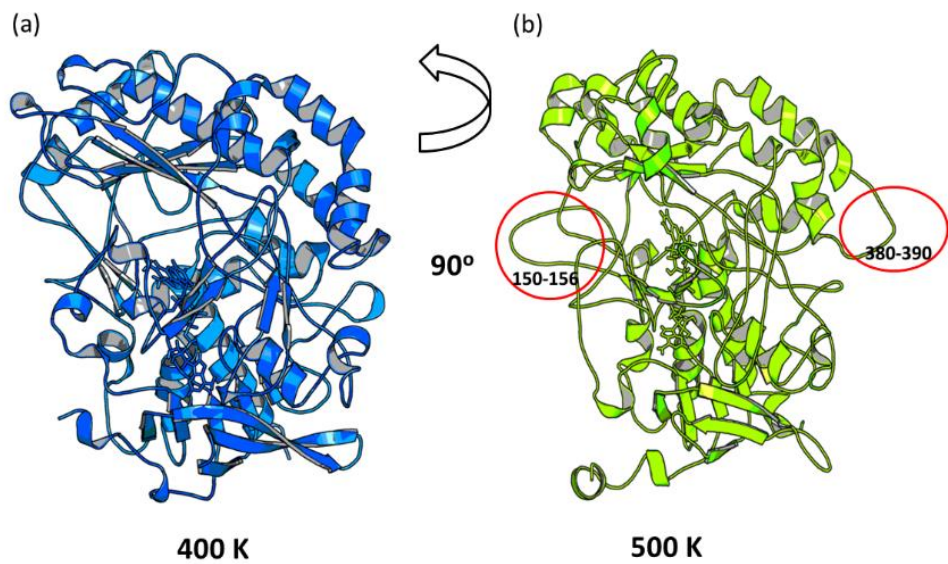

Figure 3. Final trajectory representation of the GOD_IPBCC_1CF3 structure in the form of a holoenzyme (in the cartoon). The destabilization of the structure is marked in the red circle at $500 \mathrm{~K}$.

In the secondary structure observation at 350 and $400 \mathrm{~K}$, the dynamics of each stabilizer residues group appeared similar, as indicated by the low residual mobility of the RMSF value (Figure 1b). Figure 2 shows a stable conformation of the $\alpha 4$ structure in the binding domain with FAD. This structure requires maintenance due to the functions of stabilizing the flavin 
group in FAD by forming hydrogen bonds. However, at $500 \mathrm{~K}$, a dominant structural change was identified from a decrease in the packing density of the hydrophobic core, caused by protein expansion. Partial destabilization of GOD_IPBCC_1CF3 was observed at the start of the transition time around $25 \mathrm{~ns}$ (Figure 2). This occurrence was characterized by losing the alpha-helical structure to a loop form (residues 150-156 and 380-390), leading to an irregular structure conformation (Figure 3).

\subsection{Thermostability based on salt bridge and hydrogen bond analysis.}

In this study, the salt bridge referred to the occurrence of a bond between the acidic and basic amino acid side chains, resulting in an electrostatic effect and hydrogen bonding with a distance of $3.2 \AA$ from the donor to the acceptor atom. The salt bridge-forming residue responsible for the structural stability is filtered based on the appearance with each temperature increase [28, 29].

Heat applications at 400 and $500 \mathrm{~K}$ caused the addition of residues to form 60 salt bridge pairs. Table 1 shows these components are constantly present in each simulation. Furthermore, the probability of the interaction appears very significant in increasing the protein thermal stability [30].

The results described Asp573 (D573) as the additional residue in the formation of electrostatic bonds. Also, at 350K, the OD2 atom on the carboxyl group of the D573 side chain binds to the $\mathrm{H}$ atom on the amine group of the Arg543 (R543) side chain, followed by the carbonyl OD1 atom of D573 with the amine group H atom of R535. Meanwhile, at 400K, the OD1 carbonyl atom of D573 appears oriented, without bonding electrostatically with the $\neg$ amine group of R535. This causes the ionic interaction center to shift towards the OD2 atom, but the salt bridge disintegrates at $500 \mathrm{~K}$. The interaction is formed on the two $\mathrm{H}$ atoms from individual $\mathrm{H}$ atoms of the R543 amine group with two $\mathrm{O}$ atoms of the D573 side chain to instigate an alpha-helical change into a loop structure at the $\mathrm{C}$ end region (Figure 4). The Oxygen-Nitrogen bond distance between the salt bridge connections becomes more stable with increased system temperature [31,32], indicating the salt bridge plays an important role as a contributor to the thermal stability GOD_IPBCC_1CF3.

The salt bridge pairs analyzed in the GOD_IPBCC_1CF3 structure are mostly on the surface and a small number of interior parts (core) (Table 1). The previous report shows that minimal charged residue pairs in the interior tend to stabilize the tertiary structure of GOD through the bonding between the secondary structures [33]. This interaction forms a charged residue cluster center involving D535, D571, and E282 deposits as a barrier to the trapped solvent around the FAD molecule. Also, among the observed thermostable residue pairs, a D573-mediated salt bridge network possibly serves as the main element stabilizing the $\mathrm{C}$ end position, precisely behind the interior of GOD_IPBCC_1CF3 with a system of five parallel beta-sheet structures. This beta-sheet system is reinforced by salt bridge-forming residue pairs, including E266-K250 and E38-R237, to stabilize the parallel beta-sheet system in the FADbinding domain residues E485-R470 link $\beta 8$ and $\alpha 17$ in the substrate-binding antiparallel betasheet system. However, it is necessary to increase the simulation time and temperature to observe further the thermal physiological resistance from each pair of salt bridge-forming residues when GOD_IPBCC_1CF3 is completely destabilized. 


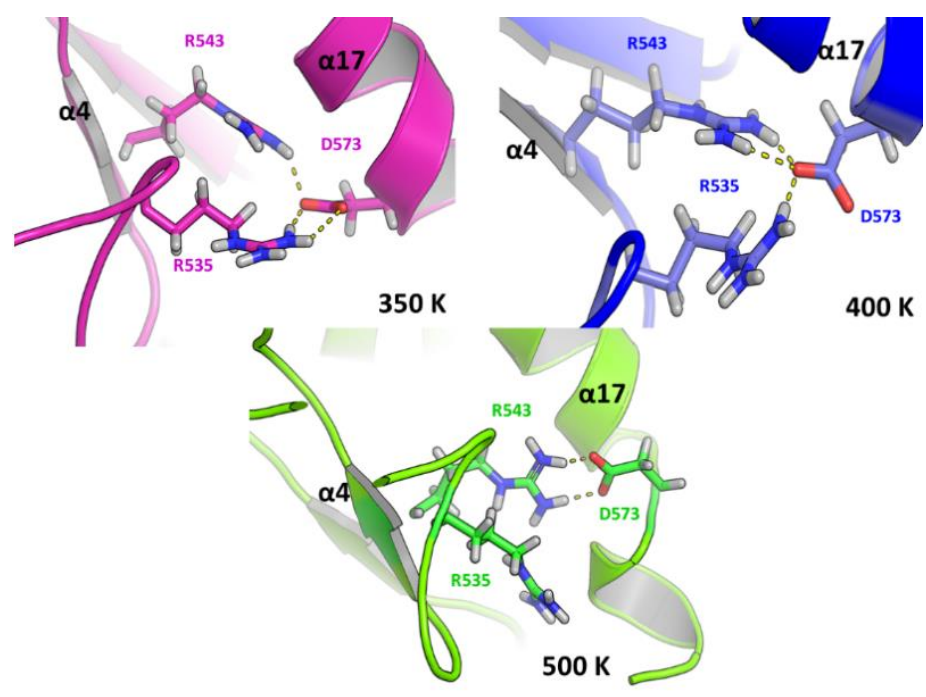

Figure 4. Comparison of connected salt bridge pairs for each temperature of the GOD_IPBCC_1CF3 structure. The dotted line (yellow) indicates the hydrogen bond.

Table 1. The final trajectory salt bridge pair, bond distance $(\AA ̊)$, and location of each stabilizing residue at different temperatures.

\begin{tabular}{l|lll|l|l|l} 
Salt Bridge Pair & $\mathbf{3 0 0 K}$ & $\mathbf{3 5 0 K}$ & $\mathbf{4 0 0 K}$ & $\mathbf{5 0 0 K}$ & Location \\
\hline R535(NH2)-D282(OD) & - & - & - & 3.25 & exterior \\
\hline D573(OD) - R533(NH2) & 3.92 & 3.87 & 3.96 & - & exterior \\
\hline D573(OD) - R543(NH2) & 4.59 & 4.53 & 4.34 & 3.45 & exterior \\
\hline E266(OE)- K250(NH2) & 2.89 & 2.83 & 3.24 & - & exterior \\
\hline E485(OE) -R470(NH2) & 3.49 & 3.54 & 2.83 & - & exterior \\
\hline E485(OE) - R335(NH2) & - & - & - & 4.70 & exterior \\
\hline D571(OD) - R35(NH2) & 3.65 & 3.30 & 3.41 & 3.66 & interior \\
\hline E282(OE) - R543(NH2) & 3.50 & 3.40 & 3.37 & 4.62 & exterior \\
\hline E38(OE)- R237(NH2) & 3.55 & 4.05 & 3.89 & 4.11 & exterior \\
\hline E142(OE) - R111(NH2) & 4.60 & 3.50 & 4.20 & 3.60 & interior
\end{tabular}

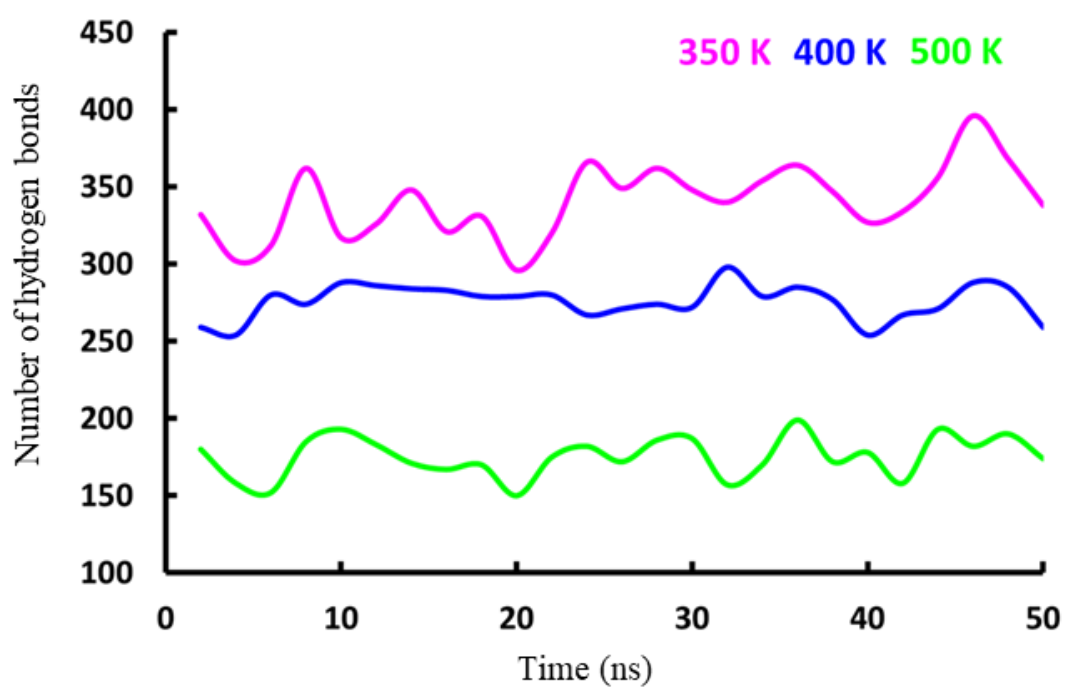

Figure 5. Profile on the number of protein-solvent hydrogen bonds from each 2 ns trajectory as a simulation time function.

The effect of water as a solvent on the movement of the GOD_IPBCC_1CF3 structure was also analyzed by changing the number of water-protein hydrogen bonds for every $2 \mathrm{~ns}$ structure across the simulation time. Figure 5 shows the average number of bonds formed was 327,265 , and 168 for temperatures of $350 \mathrm{~K}, 400 \mathrm{~K}$, and $500 \mathrm{~K}$, respectively. Furthermore, the reduced structural cohesiveness of GOD_IPBCC_1CF3 also causes the solvent polarization to decrease broadly as the temperature increased. Therefore, the intermolecular interactions of the 
protein with water are reduced. Consequently, the average percentage of this bond declined by $50 \%$ between $350-500 \mathrm{~K}$. This outcome is expected as the protein undergoes a partial unfolding stage.

\subsection{Enzyme-coenzyme stability and active site region dynamics.}

The GOD_IPBCC_1CF3 stability in performing the inherent catalytic function is influenced by coenzyme FAD presence. Figure 1 represents the dynamic structural results, where the holoenzyme's conformation appears more stable during the simulation. This stability is possibly determined by evaluating the intensity of interaction between the enzyme and coenzyme. Based on the calculations using the MMPBSA method at 350K, the intermolecular energy with residual components around the FAD-binding domain obtained a value of -43.53 $\mathrm{kcal} \mathrm{mol}^{-1}$. The strength is also influenced by the amino acids responsible for forming hydrogen and hydrophobic bonds with FAD [34-36].

Table 2. List of amino acids (residues) forming hydrogen bonds with FAD in the $350 \mathrm{~K}$ system.

\begin{tabular}{c|c|c} 
Acceptor & Donor & Occupancy $(\%)$ \\
\hline E48(OE) & FAD582(O) & 75.57 \\
\hline V248(O) & FAD582(N) & 46.92 \\
\hline FAD582(O) & S101(N) & 44.5 \\
\hline FAD582(O) & N105(N) & 20.78 \\
\hline FAD582(O) & G95(O) & 0.22
\end{tabular}

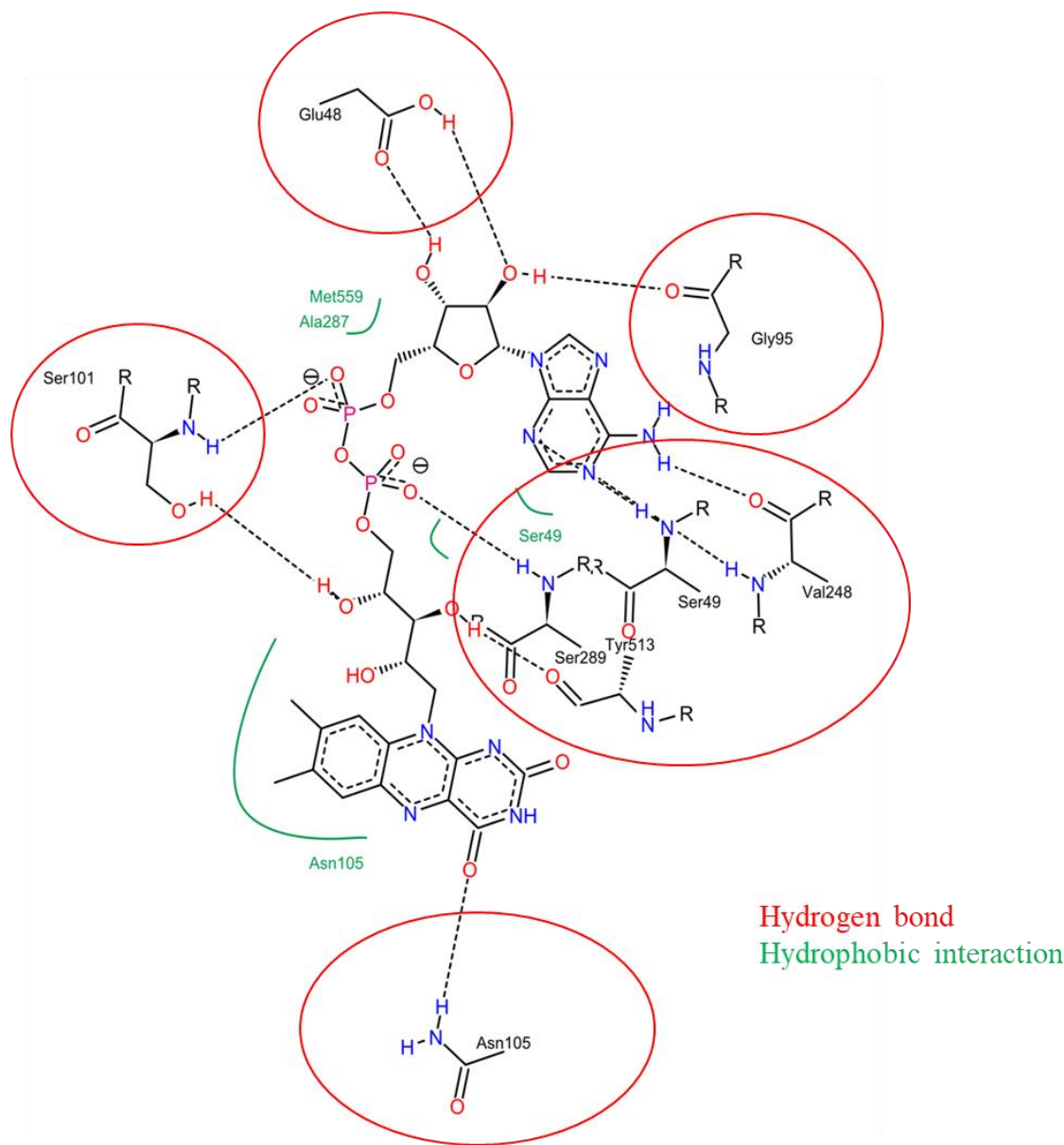

Figure 6. 2D visualization of the residual components interacting with the FAD structure.

The observation results at $350 \mathrm{~K}$ identified several amino acids capable of generating hydrogen bonds with FAD. Table 2 shows E48 as the only amino acid to experience an increase 
in the interaction percentage during the simulation with an occupancy value of $75.57 \%$. These results are consistent with previous experimental studies where E48 serves as a stabilizer residue to support two hydroxyl groups of ribose sugar as one of the constituent elements on the FAD structure [33]. Another analysis showed that one of the hydrogen atoms on the ribose hydroxyl group also forms a hydrogen bond with the carbonyl group of the G95 residue, although the formation percentage is not very significant. Furthermore, the residue component with another dominant proportion refers to residue V248, stabilizing the adenine base (46.92\%). In addition to the contribution of hydrogen bonding, certain residues are also interacting hydrophobically around the coenzyme molecular environment. However, the active center area of the isoalloxazine ring is stabilized by the side chain of the neutral polar residue N105 through the formation of hydrogen bonds and hydrophobic interactions around the ring structure. This indicates the role of N105 as a residue responsible for conformational stability as well as isoalloxazine redox reactivity (Figure 6).

The geometrical mobility of the catalytic triad (H514, H557, and E410) was observed on the basis of change in the area during the thermal stability simulation. In addition, the area calculation is based on the distance between the three residues for each system temperature. The catalytic triad area curves at 350 and $400 \mathrm{~K}$ relatively varied, with the lowest values occurring within both systems' $40 \mathrm{~ns}$ transition period. However, the residue area difference in the two systems was reportedly $26 \mathrm{~ns}$, indicating a slightly broader triad catalytic in the $400 \mathrm{~K}$ system, compared to the $350 \mathrm{~K}$. Based on the final trajectory visualization analysis, this may be due to the reorganization effect of the H516 side chain, while both $\mathrm{H} 557$ and E410 residues were relatively preserved as evidenced by the constant hydrogen bond distance (1.8 ̊́) (Figure 7).
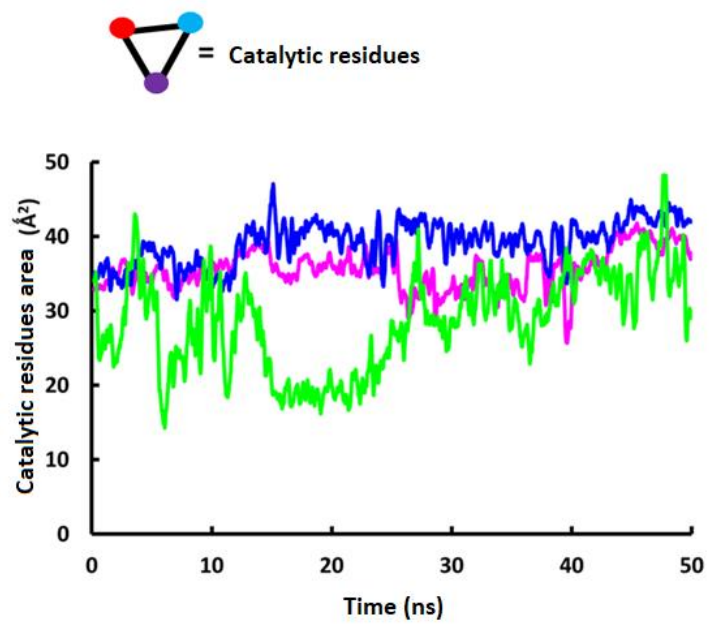

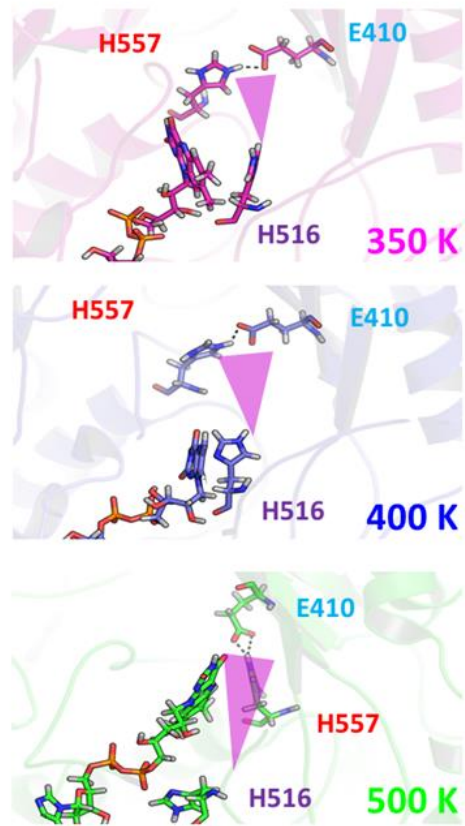

Figure 7. Area profile of the catalytic triad as a function of simulation time (ns) and the residual structure visualization of the final trajectory. The dotted black line indicates the hydrogen bond.

The system temperature increase possibly improves the flexibility characteristics of the imidazole ring from the $\mathrm{H} 516$ residue to become more distorted. This change, therefore, affects the catalytic area responsible for the impact on enzymatic rate and efficiency of the $\mathrm{z}$ enzymesubstrate bond. Furthermore, the conformational destabilization of the three catalytic residues appears more obvious as partial unfolding $(500 \mathrm{~K})$ is attained. The catalytic triad area 
experienced significant dynamics, compared to the 350 and $400 \mathrm{~K}$ systems, both from the curve and structural visualization. Gap formation (narrower) occurred at a transition period between 15-26 ns, leading to a narrowed area. This event is proportional to the increasingly irregular reorientation of the catalytic triad structure extracted from the final simulation. Despite the shift in position, the amine group of the H557 side chain forms an additional hydrogen bond with a carboxyl group of E410 to possibly minimize structural destabilization.

\section{Conclusions}

Based on the results and discussions, the thermally stabilized residues were spread over several significant segments, including D576 at the C end position, E266-R250, and E38-R237 in the FAD-binding domain E485-R470 in the substrate-binding antiparallel beta system. Therefore, the FAD molecule flexibility against temperature depends on conserve E48 by stabilizing the ribose sugar moiety.

\section{Funding}

This research was funded by the PDUPT research program, with grant number 2573/IT3.L1/PN/2020.

\section{Acknowledgments}

The authors are grateful for the financial support by the Ministry of Research and Technology/National Research and Innovation Agency of Republic Indonesia.

\section{Conflicts of Interest}

The authors declare no conflict of interest.

\section{References}

1. Samukaite-Bubniene, U.; Mazetyte-Stasinskiene, R.; Chernyakova, K.; Karpicz, R.; Ramanavicius, A. Timeresolved fluorescence spectroscopy based evaluation of stability of glucose oxidase. Int. J. Biol. Macromol. 2020, 163, 676-682, https://doi.org/10.1016/j.ijbiomac.2020.06.284.

2. Cohen, R.; Cohen, Y.; Mukha, D.; Yehezkeli, O. Oxygen insensitive amperometric glucose biosensor based on FAD dependent glucose dehydrogenase co-entrapped with DCPIP or DCNQ in a polydopamine layer. Electrochim. Acta 2021, 367, 137477, https://doi.org/10.1016/j.electacta.2020.137477.

3. Indriani, A.; Ambarsari, L.; Zilda, D.S. Purification and Characterization of Glucose Oxidase of Aspergillus niger IPBCC 08.610. Microbiol. Indones. 2018, 12, 43-48, https://doi.org/10.5454/mi.12.2.2.

4. Rohmayanti T, Ambarsari L, Maddu A. Enzymatic activityof glucose oxidase from Aspergillus niger IPBCC. 08.610 on modified carbon paste electrode as glucose biosensor. IOP Conf. Ser.: Earth Environ. Sci. 2017, 58, 012046. https://doi.org/10.1088/1755-1315/58/1/012046.

5. Ambarsari, L.; Setyawati, I.; Kurniasih, R.; Kurniatin, P.A.; Maddu, A. Immobilization of glucose oxidase on modified-carbon-paste-electrodes for microfuel cell. Indones. J. Chem. 2016, 16, 92-97, https://doi.org/10.22146/ijc.21183.

6. Maulana, F.A.; Ambarsari, L.; Wahyudi, S.T. Homology Modeling and Structural Dynamics of the Glucose Oxidase. Indones. J. Chem. 2020, 20, 43-53, https://doi.org/10.22146/ijc.39135.

7. Kurniatin, P.A.; Ambarsari, L.; Khanza, A.D.A.; Setyawati, I.; Seno, D.S.H.; Nurcholis, W. Characteristics of Glucose Oxidase Gene (GGOx) from Aspergillus niger IPBCC 08.610. Jurnal Kimia Valensi 2020, 6, 1019, https://doi.org/10.15408/jkv.v6i1.9440.

8. Biasini, M.; Bienert, S.; Waterhouse, A.; Arnold, K.; Studer, G.; Schmidt, T.; Kiefer, F.; Cassarino, T.G.; Bertoni, M.; Bordoli, L.; Schwede, T. SWISS-MODEL: Modelling protein tertiary and quaternary structure 
using evolutionary information. Nucleic Acids Res. 2014, 42, W252-W258, https://doi.org/10.1093/nar/gku340.

9. Amber 2016 Reference Manual (Cover Amber16 and amberTools16). https://ambermd.org/doc12/Amber16.pdf.

10. Magis, C.; Taly, J.F.; Bussotti, G.; Chang, J.M.; Di Tommaso, P.; Erb, I.; Espinosa-Carrasco, J.; Notredame, C. T-coffee: Tree-based consistency objective function for alignment evaluation. Methods Mol. Biol. 2014, 1079, 117-129, https://doi.org/10.1007/978-1-62703-646-7_7.

11. Anandakrishnan, R.; Aguilar, B.; Onufriev, A.V. H++ 3.0: Automating pK prediction and the preparation of biomolecular structures for atomistic molecular modeling and simulations. Nucleic Acids Res. 2012, 40, W537-W541, https://doi.org/10.1093/nar/gks375.

12. Gasteiger, E.; Hoogland, C.; Gattiker, A.; Duvaud, S.; Wilkins, M.R.; Appel, R.D.; Bairoch, A. Protein Identification and Analysis Tools on the ExPASy Server. In: Walker J.M. (eds) The Proteomics Protocols Handbook. Springer Protocols Handbooks. Humana Press. 2005, 571-607, https://doi.org/10.1385/1-59259890-0:571.

13. Laskowski, R.A.; Jabłońska, J.; Pravda, L.; Vařeková, R.S.; Thornton, J.M. PDBsum: Structural summaries of PDB entries. Protein Sci. 2018, 27, 129-134, https://doi.org/10.1002/pro.3289.

14. Laskowski, R.A; PDBsum: summaries and analyses of PDB structures. Nucleic Acids Res. 2001, 29, 221222, https://doi.org/10.1093/nar/29.1.221.

15. Chen, V.B.; Arendall, W.B.; Headd, J.J.; Keedy, D.A.; Immormino, R.M.; Kapral, G.J.; Murray, L.W.; Richardson, J.S.; Richardson, D.C. MolProbity: All-atom structure validation for macromolecular crystallography. Acta Cryst. 2010, 66, 12-21, https://doi.org/10.1107/S0907444909042073.

16. Wallner, B.; Elofsson, A. Can correct protein models be identified?. Protein Sci. 2003, 12, 1073-1086, https://doi.org/10.1110/ps.0236803.

17. Roe, D.R.; Cheatham, T.E.; PTRAJ and CPPTRAJ: Software for processing and analysis of molecular dynamics trajectory data. J. Chem. Theory Comput. 2013, 9, 3084-3095, https://doi.org/10.1021/ct400341p.

18. Miller, B.R.; McGee, T.D.; Swails, J.M.; Homeyer, N.; Gohlke, H.; Roitberg, A.E. MMPBSA.py: An efficient program for end-state free energy calculations. J. Chem. Theory Comput. 2012, 8, 3314-3321, https://doi.org/10.1021/ct300418h.

19. Reetz, M.T.; Carballeira, J.D.; Vogel, A. Iterative saturation mutagenesis on the basis of b factors as a strategy for increasing protein thermostability. Angew. Chem. Int. Ed. 2006, 45, 7745-7751, https://doi.org/10.1002/anie.200602795.

20. Finn, R.D.; Coggill, P.; Eberhardt, R.Y.; Eddy, S.R.; Mistry, J.; Mitchell, A.L.; Potter, S.C.; Punta, M.; Qureshi, M.; Sangrador-Vegas, A.; Salazar, G.A.; Tate, J.; Bateman, A. The Pfam protein families database: Towards a more sustainable future. Nucleic Acids Res. 2016, 44, D279-D285, https://doi.org/10.1093/nar/gkv1344.

21. Humphrey, W.; Dalke, A.; Schulten, K.; VMD.Visual molecular dynamics. J. Mol. Graph. 1996, 33-38, https://doi.org/10.1016/0263-7855(96)00018-5.

22. Zarezade, V.; Rezaei, H.; Shakerinezhad, G.; Safavi, A.; Nazeri, Z.; Veisi, A.; Azadbakht, O.; Hatami, M.; Sabaghan, M.; Shajirat, Z. The identification of novel inhibitors of human angiotensin-converting enzyme 2 and main protease of Sars-Cov-2: A combination of in silico methods for treatment of COVID-19. J. Mol. Struct. 2021, 1237, 130409, https://doi.org/10.1016/j.molstruc.2021.130409.

23. Zakeri, A.; Khoshsorour, S.; Fath, M.K.; Pourzardosht, N.; Fazeli, F.; Khalili, S. Structural analyses and engineering of the pmHAS enzyme to improve its functional performance: An in silico study. J. Carbohydr. Chem.. 2020, 39, 354-373, https://doi.org/10.1080/07328303.2020.1821041.

24. Ma, H.; Zou, T.; Li, H.; Cheng, H. The interaction of sodium dodecyl sulfate with trypsin: Multi-spectroscopic analysis, molecular docking, and molecular dynamics simulation. Int. J. Biol. Macromol. 2020, 162, 15461554, https://doi.org/10.1016/j.ijbiomac.2020.08.020.

25. Parida, P.; Bhowmick, S.; Saha, A.; Islam, M.A. Insight into the screening of potential beta-lactamase inhibitors as anti-bacterial chemical agents through pharmacoinformatics study. J. Biomol. Struct. Dyn. 2021, 39, 923-942, https://doi.org/10.1080/07391102.2020.1720819.

26. Biswas, P.; Adhikari, A.; Pal, U.; Singh, P.; Das, M.; Saha-Dasgupta, T.; Choudhury, S.S.; Das, R.; Pal, S.S. Flexibility modulates the catalytic activity of a thermostable enzyme: key information from optical spectroscopy and molecular dynamics simulation. Soft Matter 2020, 16, 3050-3062, https://doi.org/10.1039/C9SM02479D. 
27. Sharma, J.; Bhardwaj, V.K.; Singh, R.; Rajendran, V.; Purohit, R.; Kumar, S. An in-silico evaluation of different bioactive molecules of tea for their inhibition potency against non structural protein-15 of SARSCoV-2. Food Chem. 2021, 346, 128933, https://doi.org/10.1016/j.foodchem.2020.128933.

28. Ban, X.; Wu, J.; Kaustubh, B.; Lahiri, P.; Dhoble, A.S.; Gu, Z.; Li. C.; Cheng, L.; Hong, Y.; Tong, Y.; Li, Z. Additional salt bridges improve the thermostability of 1,4- $\alpha$-glucan branching enzyme. Food Chem. 2020, 316, 126348, https://doi.org/10.1016/j.foodchem.2020.126348.

29. Son, H.; Seo, H.; Han, S.; Kim, S.M.; Pham, L.T.M.; Khan, M.F.; Sung, H.J.; Kang, S.; Kim, K.; Kim, Y.H. Extra disulfide and ionic salt bridge improves the thermostability of lignin peroxidase H8 under acidic condition. Enzyme Microb. Technol. 2021, 148, 109803, https://doi.org/10.1016/j.enzmictec.2021.109803.

30. Chen, L.; Li, X.; Wang, R.; Fang, F.; Yang, W.; Kan, W. Thermal stability and unfolding pathways of hyperthermophilic and mesophilic periplasmic binding proteins studied by molecular dynamics simulation. J. Biomol. Struct. Dyn. 2016, 34, 1576-1589, https://doi.org/10.1080/07391102.2015.1084480.

31. Hait, S.; Mallik, S.; Basu, S.; Kundu, S. Finding the generalized molecular principles of protein thermal stability. Proteins 2020, 88, 788-808, https://doi.org/10.1002/prot.25866.

32. Bureau, H.; Quirk, S.; Hernandez, R.; The relative stability of trpzip1 and its mutants determined by computation and experiment. RSC Adv. 2020, 10, 6520-6535, https://doi.org/10.1039/D0RA00920B.

33. Hecht, H.J.; Kalisz, H.M.; Hendle, J.; Schmid, R.D.; Schomburg, D. Crystal structure of glucose oxidase from Aspergillus niger refined at $2 \cdot 3 \AA$ reslution. J. Mol. Biol. 1993, 229, 153-172, https://doi.org/10.1006/jmbi.1993.1015.

34. Rao, Y.; Li, S.L.; Li, M.J.; Cui, S.; Gou, K.M. A single amino acid substitution in the FAD-binding domain causes the inactivation of Propionibacterium Acnes isomerase. Biosci. Biotechnol. Biochem. 2020, 84, 789796, https://doi.org/10.1080/09168451.2019.1709960.

35. Ullah, A. Structure-function studies and mechanism of action of snake venom L-amino acid oxidases. Front. Pharmacol. 2020, 11, 110, https://doi.org/10.3389/fphar.2020.00110.

36. Masakari, Y.; Hara, C.; Araki, Y.; Gomi, K.; Ito, K. Improvement in the thermal stability of Mucor prainiiderived FAD-dependent glucose dehydrogenase via protein chimerization. Enzyme Microb. Technol. 2020, 132, 109387, https://doi.org/10.1016/j.enzmictec.2019.109387. 